\title{
INFRARED LINE EMISSION BY BROADLY DISTRIBUTED GAS
}

\author{
Martin Harwit \\ Laboratory for Astrophysics \\ National Air and Space Museum \\ Smithsonian Institution \\ Washington, DC 20560 USA
}

\begin{abstract}
Diffuse infrared background radiation measurements have traditionally been carried out across broad wavelength bands, primarily because there seldom appeared to be sufficient energy available in individual spectral lines to make such observations possible. In the past few years, far-infrared searches for spectral line background radiation have become possible, and considerably greater use of these capabilities will become available in the next few years. The promise of this kind of observation is reviewed.
\end{abstract}

\section{INTRODUCTION}

Infrared spectral line observations carried out in recent years have shown that certain lines can be extremely luminous. Among these the far-infrared fine-structure lines of singly ionized carbon and neutral oxygen are some of the most prominent features in neutral atomic hydrogen clouds and are also strong at the interfaces of ionized and molecular regions, while doubly ionized oxygen lines are a reliable indicator of ionized nebulosity.

The strength of this radiation within the Galaxy has been difficult to ascertain, because we are embedded in and surrounded by regions emitting at various surface brightness levels. An absolute zero level for surface brightness has therefore been difficult to establish in all but a few exceptional cases. On the other hand, extragalactic observations have shown that line emission from galaxies can amount to a substantial fraction of a percent of the total luminosity in the strongest spectral lines. From such measurements on spiral galaxies, we can infer emission at comparable levels from the Milky Way. That emission should become observable with the coming generation of space observatories, including the Cosmic Background Explorer (COBE), the Infrared Space Observatory (ISO), the Submillimeter Wavelength Astronomical Satellite (SWAS), and the Space Infrared Telescope Facility (SIRTF). A number of other, shorter-duration rocket or satellite missions will also make contributions to our understanding.

While the existence of a galactic diffuse background at individual line frequencies is well known, though still not well studied, we have no idea whether spectral features exist in the diffuse extragalactic background. We may expect that such features will be prevalent if stages of luminous line emission existed at well-defined and cosmically well-synchronized epochs in the evolution of quasars or galaxies. One possible instance of luminous line emission could be expected during protogalactic collapse, where cooling through collisional excitation of the $v=1$ vibrational level of molecular hydrogen followed by radiative cooling of the gas through $v=1$ to $v=0$ rovibrational transitions would result in an emission peak at wavelengths around $2 \mu \mathrm{m}$ in the protogalaxy's rest frame. Provided a predominant fraction of all galaxies passed through such a ubiquitous, well-synchronized, sharply defined epoch centered on redshift $z$, a pronounced emission peak should be detectable at wavelengths $2(z+1) \mu \mathrm{m}$. Collisionally 
excited rotational states of the ground vibrational level would be discerned at correspondingly longer wavelengths (cf. Lepp and Schull, 1984). Future observations will have to be awaited to see whether such precisely synchronized short-lived eras of intense line emission ever took place.

The purpose of this paper is to discuss a selection of observations obtained to date, as well as promising projects that might be undertaken in the future to learn as much as possible from diffuse line emission in the infrared. No attempt is made to discuss this question exhaustively. Rather, the aim of the paper is to call attention to and provide an informal overview of the promise of this field.

\section{DIFFUSE EMISSION FROM HII REGIONS}

Diffuse infrared emission from HII regions in the Galaxy has been known for over a decade. Early mid-infrared ground-based observations detected the $\mathrm{Ne}^{+}$line at $12.8 \mu \mathrm{m}$. Later, airborne infrared observations at longer wavelengths became possible in the mid-1970s and rapidly established strong line emission of $\mathrm{O}^{++}$at 88.4 and $51.8 \mu \mathrm{m}$, from atomic oxygen, $\mathrm{O}$, at 63 and $145.5 \mu \mathrm{m}$, from $\mathrm{S}^{++}$at 18.7 and $33.5 \mu \mathrm{m}$, from $\mathrm{N}^{++}$at $57.3 \mu \mathrm{m}$, and from $\mathrm{C}^{+}$at $157.7 \mu \mathrm{m}$.

Some of the strongest of these lines have luminosities amounting to a substantial fraction of $1 \%$ of the total far-infrared emission of an HII region complex. To the extent that this line emission tells one a great deal about local abundances, densities, temperatures, and states of ionization, observations of such lines can be more informative than continuum observations in the far-infrared, which primarily convey a measure of the radiation energy budget of such a complex, although they carry chemical or mineralogical information as well.

By no means all of this observed line radiation is emitted from the ionized gas. Some of the $\mathrm{C}^{+}$and $\mathrm{O}$ emission comes from adjacent, diffuse neutral hydrogen regions and from atomic clouds, as well as from shocked and photodissociation regions. And while the $\mathrm{O}^{++}, \mathrm{N}^{++}$, and $\mathrm{S}^{++}$as well as $\mathrm{C}^{+}$emission may originate in well-defined HII regions, a certain fraction also appears to emanate from extended low density ionized regions.

\section{ABSOLUTE SPECTRAL LINE SURFACE BRIGHTNESS IN THE INFRARED}

Absolute background spectral line radiation measurements are difficult to obtain, particularly at infrared wavelengths where the atmosphere strongly emits. Satellite observations are the ultimate answer to that problem, but to date no satellite observatory has had sufficient spectral resolution to observe broadly distributed spectral line emission.

The first attempt at a detection of the absolute level of far-infrared diffuse line emission was carried out by Stacey et al. (1983) (see also Stacey, 1985), who used a lunar occultation technique to obtain surface brightness measurements in the $\mathrm{C}^{+}$line. In an experiment timed to coincide with the passage of the Moon through the galactic plane, the observers compared radiation levels obtained from the limb of the Moon to a region of the sky just off the limb. Carried out from the NASA Lear jet, these observations, obtained by chopping between sky and lunar limb, demonstrated the existence of considerable $\mathrm{C}^{+}$emission near the galactic plane and, at the limited level of sensitivity of the observations, no measurable emission at higher galactic latitudes. The duration of the most critical of these observations was of the order of half an hour, during which time the Moon moved about $11^{\prime}$ perpendicular to the plane, while also 
moving about $6^{\prime}$ along the plane. By taking similar measurements on other days, when the Moon was at higher galactic latitudes, a comparison of galactic plane emission with surface brightness off the plane was obtained. The observed spectral-line surface brightness at close approach to the plane, in a region $b^{\mathrm{II}}=-0.5$ to $-7.5^{\prime}$ at $l^{\mathrm{II}}=8^{\circ}$ was $-5 \times 10^{-2} \mathrm{erg} \mathrm{s}^{-1} \mathrm{~cm}^{-2} \mathrm{sr}^{-1}$, comparable with the surface brightness measured from HII regions. A similarly long strip centered at $b^{\text {II }} \sim-14^{\prime}$ was compatible with zero emission within the error bars.

\section{4. $\mathrm{C}^{+}$EMISSION FROM THE GALAXY}

Because the line emission observed in the lunar occultation experiment appeared to be so narrowly confined to the galactic plane, later observations from the NASA Lear jet were carried out through a chopping technique in which the beam was successively stepped across the galactic plane (Stacey, 1985; Stacey et al., 1985). This more flexible technique yielded further profiles of galactic plane emission, with peak surface brightness more like 1 to $2 \times 10^{-3}$ erg $\mathrm{s}^{-1} \mathrm{~cm}^{-2} \mathrm{sr}^{-1}$ and a width of $0.5-1^{\circ}$ for the galactic plane. Those results suggest that the earlier lunar-occultation galactic-plane-crossings may have transited a region of rather high Galaxy surface brightness.

More recent balloon observations by Shibai et al. (1989) report emission of comparably high surface brightness at $l^{\mathrm{II}} \sim 31^{\circ}$ near W43, but generally rather lower levels around $2 \times 10^{-4}$ $\mathrm{erg} \mathrm{s}^{-1} \mathrm{~cm}^{-2} \mathrm{sr}^{-1}$ at most positions between $l^{\mathrm{II}}=30^{\circ}$ and $50^{\circ}$. Their width for the plane is of order $1-2^{\circ}$. According to Okuda et al. (1989), for regions between $l^{\mathrm{II}}=-40^{\circ}$ and $+40^{\circ}$, the signal varies from $\sim 5 \times 10^{-4}$ to $2 \times 10^{-3} \mathrm{erg} \mathrm{s}^{-1} \mathrm{~cm}^{-2} \mathrm{sr}^{-1}$ in a $3.6^{\prime}$ square field of view chopped $19^{\prime}$ primarily along a direction crossing the galactic plane.

Shibai et al. (1989) find the total $\mathrm{C}^{+}$line emission at $l^{\mathrm{II}}=30^{\circ}$ to $50^{\circ}$ to correspond to $0.1-0.5 \%$ of the total IRAS emission. We will see below that this measurement compares favorably with observations from extragalactic sources.

\section{POSSIBLE ATOMIC OXYGEN EMISSION AT $63 \mu \mathrm{m}$}

One of the most striking early results from the Infrared Astronomical Satellite (IRAS) was the diffuse far-infrared emission at high galactic latitudes in the 60 and $100 \mu \mathrm{m}$ bands (Hauser et al., 1984; Low et al., 1984). The possibility that some fraction of this radiation might be due to atomic oxygen emission at $63 \mu \mathrm{m}$ or $\mathrm{O}^{++}$emission at 51.8 and $88.4 \mu \mathrm{m}$ was raised by Harwit, Houck, and Stacey (1986). To date no direct observations of that line radiation have been possible, and gas densities and temperatures at high galactic latitudes are sufficiently uncertain that no reliable estimate of the emission by atoms and ions, following collisional excitation, are possible. However, collisional excitation of atomic oxygen, followed by radiative deexcitation, is an effective way of cooling gas - atom for atom considerably more effective than the cooling that could be provided by the same amount of heavy atomic material condensed in grains or polyaromatic hydrocarbons (PAHs).

If we take the collisional excitation cross section of an atom to be of order $10^{-16} \mathrm{~cm}^{2}$, and consider grains to be roughly spherical, with interatomic distances of order $10^{-8} \mathrm{~cm}$ and a hydrogen-to-heavy atom ratio, by number, to be $1: 1$, we can assume that impinging atoms deposit all of their energy in each grain, while transferring only $10^{-2} \mathrm{eV}$ to an atom of oxygen at each collision. This will produce a cooling rate per atom, $L$, that can be expressed in terms of the product of an effective collisional cross section, $s$, per atom, and of the amount, $e$, of 
collisional energy actually dissipated in the collision. These rates are shown in Table 1 for gas velocities, respectively, of 1 and $10 \mathrm{~km} \mathrm{~s}^{-1}$.

TABLE 1. Relative Effectiveness of Excitation

\begin{tabular}{lcc}
\hline \multicolumn{1}{c}{ Species } & $\mathrm{v}=10 \mathrm{~km} \mathrm{~s}^{-1}$ & $\mathrm{v}=1 \mathrm{~km} \mathrm{~s}^{-1}$ \\
\hline [OI] & $2 \times 10^{-18} \mathrm{eV} \mathrm{cm}^{2}$ & $2 \times 10^{-18} \mathrm{eV} \mathrm{cm}^{2}$ \\
$10^{-5} \mathrm{~cm}$ grain & $10^{-19}$ & $10^{-21}$ \\
$10^{-7} \mathrm{~cm} \mathrm{PAH}$ & $10^{-17}$ & $10^{-19}$ \\
\hline
\end{tabular}

Deul (1988) has found a correlation between HI density at high galactic latitudes and 10 $\mu \mathrm{m}$ brightness corrected for zodiacal light emission. He also found that some of the atomic hydrogen velocity distributions at the peak of the far-infrared emission show velocity dispersions of order $10 \mathrm{~km} \mathrm{~s}^{-1}$, though this may be exceptional. At any rate, Table 1 takes into account cooling rates both in conventional, low-temperature gases and in gas where atomic hydrogen would most probably be observed undergoing shocks. While the fraction of heavy elements in atomic form, as contrasted to the fraction embedded in grains, is not known, it appears that atomic cooling at $63 \mu \mathrm{m}$ should play a fairly prominent role, since PAHs are not considered likely to be producing significant $100 \mu \mathrm{m}$ emission.

\section{FAR-INFRARED LINE EMISSION FROM EXTERNAL GALAXIES}

In a recent review, Stacey (1989) provides $\mathrm{C}^{+}$emission data on 19 external galaxies. The ratio of $\mathrm{C}^{+}$line emission to total far-infrared emission ranges from $<10^{-3}$ for the peculiar galaxy NGC 660 to $1.5 \times 10^{-2}$ for M51. Interestingly, Shibai et al. (1989) find the galactic emission between $l^{\mathrm{II}}=30^{\circ}$ and $50^{\circ}$ to lie comparably in the range $10^{-3}$ to $5 \times 10^{-3}$ of the total IRAS infrared emission. This seems to be a prevalent trait.

The $\mathrm{C}^{+}$emission from M82 exhibits a central surface brightness of $1.5 \times 10^{-3} \mathrm{erg}$ $\mathrm{s}^{-1} \mathrm{~cm}^{-2} \mathrm{sr}^{-1}$, corresponding to a luminosity $L\left(C^{+}\right)=4.4 \times 10^{7} L_{\odot}$, with $L(F I R)=2.8 \times 10^{10} L_{\odot}$ For NGC 1068, $L\left(C^{+}\right)=6.4 \times 10^{8} L_{\odot}$, while $L(F I R)=1.15 \times 10^{11} L_{\odot}$. The ultraluminous galaxy NGC 6240 with $L(F I R)=6.2 \times 10^{11} L_{\odot}$ has $L\left(C^{+}\right)=9 \times 10^{8} L_{\odot}$. In all of these sources, varying from relatively normal galaxies like ours, to strong infrared emitters like M82, Seyfert galaxies like NGC 1068, and ultraluminous galaxies like NGC 6240 , the ratio of $\mathrm{C}^{+}$emission to integrated far-infrared emission appears to extend over a narrow range, despite the wide variety of physical conditions believed responsible for the far-infrared flux.

For some galaxies, a number of different FIR lines have been observed, as Stacey (1989) points out. In particular, for M82 the $\mathrm{S}^{++}$lines at 18.7 and $33.48 \mu \mathrm{m}, \mathrm{Si}^{+}$at $34.8 \mu \mathrm{m}, \mathrm{O}^{++}$at 51.8 and $88.36 \mu \mathrm{m}, \mathrm{N}^{++}$at $57.33 \mu \mathrm{m}$, atomic $\mathrm{O}$ at 63.18 and $145.5 \mu \mathrm{m}$, and $\mathrm{C}^{+}$at $157.7 \mu \mathrm{m}$ all have been observed. The future for extragalactic observations therefore is promising, and by virtue of the similarity of many external galaxies to ours, we may expect to obtain insight into the diffuse components within the Milky Way by observing and mapping the distributed radiation in external systems. 


\section{POSSIBLE NEAR-INFRARED EMISSION FROM HIGHLY IONIZED GASES}

Fine-structure transitions in the ground states of atoms and ions are observed in the farinfrared, in the emission detected from HII regions, planetary nebulae, and cooler diffuse domains. As the gas temperature rises, however, and increasingly high states of ionization are observed, such emission lines are progressively shifted toward shorter wavelengths. Recently, a number of fine-structure transitions of highly ionized species have been observed in the emission from novae (Greenhouse et al., 1989). But these or other, similarly excited lines should be emitted also in the extremely hot gases surrounding the central galaxies in clusters. The $\mathrm{X}$-ray emission from those regions attests to million-degree temperatures at which we would expect line radiation from $\mathrm{Al}[\mathrm{IX}]$ at $2.04 \mu \mathrm{m}, \mathrm{Mg}$ [VIII] at $3.02 \mu \mathrm{m}$ and $\mathrm{Si}[\mathrm{IX}]$ at $3.92 \mu \mathrm{m}$, all observed in novae, as well as from more highly ionized species. Similar emission would be expected from giant bubbles of million-degree gases which may pervade wide realms of the Galaxy. That emission from the Galaxy still is enigmatic, as recently discussed by Kahn and Jakobsen (1988), and near infrared spectral line observations might provide additional clues to its nature.

We may also expect fine-structure emission from highly ionized species in some extragalactic sources. An order of magnitude extrapolation to the emission that might be expected from M87 on the basis of data collected by Greenhouse et al. on Nova Vulpeculae 1984 No. 2 (QU Vul) can serve as an indicative guide.

The total flux received from two sources at one and the same temperature should be identical if the product $r^{3} n_{e} n_{Z} R^{-2}$ is identical for the two, where $r$ is the radius of the source, $n_{e}$ the electron number density, $n_{Z}$ the number density of the ion $\mathrm{Z}$, and the distance to the source is $R$. This is equivalent to stating that the two sources will provide identical fluxes if the function $F=M_{Z} n_{E} R^{-2}$ is the same for the two, where $M_{Z}$ is the total mass of ions $Z$ residing in the luminous plasma.

Greenhouse et al. give $R=3.6 \mathrm{kpc}, n_{e}=2 \times 10^{6} \mathrm{~cm}^{-3}$ and $r=2 \times 10^{16} \mathrm{~cm}$ for QU Vul. We can take $M_{Z}$ to be a fraction $Z$ of the total mass $M$, which we may call $Z M$. The mass $M$ can be determined from the electron number density and radius, on the assumption that most of the material has equal numbers of protons and neutrons in the nucleus and that most of the abundant elements are fully ionized. That gives $M=10^{32} \mathrm{~g}$. The hydrogen content of this source cannot be very high, as confirmed by the low line strengths of the Brackett alpha and gamma lines. We may not be far off, in order of magnitude, if we choose $Z=10^{-2}$. On that basis $F \sim 4 \times 10^{-8}$ in cgs units.

Similarly, for M87, on the assumption that the fraction of the abundant ion is $2.6 \times 10^{-5}$ by number, comparable to the cosmic abundance of magnesium, taking the mass of hot gas enclosed by a radius of $30 \mathrm{kpc}$ to be $3 \times 10^{13} \mathrm{M} \odot$ as suggested by the X-ray data, and assuming a distance of $13 \mathrm{Mpc}$ to the source, we can compute a mean value of $n_{e}$ and obtain $F \sim 10^{-8}$ in the same cgs units. While this comparison suggests that the diffuse radiation in one of the fine-structure lines from an abundant ion should be detectable in M87, it neglects taking into account the far higher temperature, $T \sim 3 \times 10^{7} \mathrm{~K}$, cited by Tucker and Rosner (1983) for this source. As Edlen's $(1969,1972)$ papers attest, the fine-structure lines for the most highly ionized species in an isoelectronic sequence generally shift toward shorter wavelengths and no longer appear in the infrared at such high temperatures. However, exceptions to that case exist, and one of these might be the ${ }^{3} P_{1}$ to ${ }^{3} P_{0}$ transition of Fe XIX which, according to Kaufman and Sugar (1986), lies at 0.7045 . Though substantially less abundant, Cr XVII in the same isoelectric sequence has a similar transition at $4.3 \mu \mathrm{m}$. 


\section{OTHER DIFFUSE ATOMIC, IONIC, AND MOLECULAR LINE EMISSION}

Extended emission from molecular hydrogen $\mathrm{H}_{2}$ has been known to be emitted by galactic molecular clouds for some time and has been observed in several extragalactic sources also. Highly excited rotational transitions from $\mathrm{CO}$ molecules, initially discovered by the group of Charles Townes at the University of California at Berkeley, have been known for some years also. Recently Schmid-Burgk et al. (1989) have found that the $J=7-6$ transitions of CO are emitted over an extended region, roughly $10^{\prime}$ in diameter in the Orion molecular cloud OMC- 1 . Presumably, other lines of $\mathrm{CO}$, in other sources, are extended as well. Extended emission roughly proportional to $\mathrm{CO}(J=1-0)$ emission has also been traced for galactic atomic carbon, $\mathrm{C}$, by the California Institute of Technology group of Thomas Phillips. And possibly $\mathrm{Si}^{+}$ observed at $34.8 \mu \mathrm{m}$ will also be found to be extended.

\section{CONCLUSION}

A variety of ions, atoms and molecules have been observed to emit infrared line radiation over extended regions in the Galaxy as well as in extragalactic sources. We may confidently assume that, as increasingly sensitive techniques for the detection of diffuse spectral line radiation are developed, these lines will be widely detected and will provide interesting new information on the Galaxy and universe.

Acknowledgment-The author's work is supported through NASA grant NAGW-1261. He is grateful to Dr. Matthew A. Greenhouse for providing a preprint of his paper before publication and for extensive discussion on near-infrared fine-structure line emission. Similarly he is pleased to acknowledge receiving preprints of the quoted papers by Okuda et al. and Shibai et al. from Prof. H. Okuda, before those articles were published. He is greatly indebted to Dr. Yoel Rephaeli for an important critical comment.

\section{REFERENCES}

Deul, E. R. 1988, in Comets to Cosmology, A. Lawrence, ed., Lecture Notes in Physics 297, Springer Verlag, 89.

Edlen, B. 1969, Solar Physics, 9, 439.

Edlen, B. 1972, Solar Physics, 24, 356.

Greenhouse, M. A., Grasdalen, G. L., Woodward, C. E., Benson, J., Gehrz, R.D., Rosenthal, E., and Skrutskie, M. F. 1989, "The Infrared Coronal Lines of Recent Novae," to be published in Ap. J.

Harwit, M., Houck, J. R., and Stacey, G. J. 1986, Nature, 319, 646.

Hauser, M. G., et al. 1984, Ap. J., 278, L15.

Kahn, S. M., and Jakobsen, P. 1988, Ap. J., 329, 406.

Kaufman, V., and Sugar, J. 1986, J. Phys. Chem. Ref. Data, 16, 418.

Lepp, S., and Shull, M. 1984, Ap. J., 280, 465.

Low, F. J., et al. 1984, Ap. J., 278, L19.

Okuda, H., Shibai, H., Nakagawa, T., Matsuhara, H., Maihara, T., Mizutani, K., Kobayashi, Y., Hiromoto, N., Low, F. J., and Nishimura, T. 1989, "Observation of Diffuse CII Emission in the galactic Center Region," preprint.

Schmid-Burgk, J., Densing, R., Kruegel, E., Nett, H., Roeser, H.P., Schaefer, F., Schwaab, G., van der Wal, P., and Wattenbach, R. 1989, $A \& A, 215,150$.

Shibai, H., Okuda, H., Nakagawa, T., Matsuhara, H., Maihara, T., Mizutani, K., Kobayashi, Y., Hiromoto, N., Low, F. J., and Nishimura, T. 1989, "Detection of Diffuse [CII] 158 Micron Emission Along the Galactic Plane," preprint.

Stacey, G. J., Smyers, S. D., Kurtz, N. T., and Harwit, M., 1983, Ap. J., 268, L99.

Stacey, G. J. 1985, Ph.D. thesis, Cornell University.

Stacey, G. J., Viscuso, P. J., Fuller, C. E., and Kurtz, N. T. 1985, Ap. J. 289, 803. 
Stacey, G. J. 1989, in Proceedings of the 22nd ESLAB Symposium: Infrared Spectroscopy in Astronomy, ed. M.F. Kessler et al. (Noordwijk: ESA), to be published.

Tucker, W. H., and Rosner, R. 1983, Ap. J., 312, 547.

G. Münch: The observation of highly ionized species for diagnostics of the hot (coronal) gas component of the interstellar medium would be of great importance. We should recall, however, that between $10^{6}$ and $10^{4} \mathrm{~K}$ the cooling term of the interstellar medium is extremely steep, and that probably the ions at intermediate temperatures would have low abundance. The observations of [SiX] and [Mg VIII] that I made with Neugebauer and McCammon in the solar corona showed them to have a strength lower than expected.

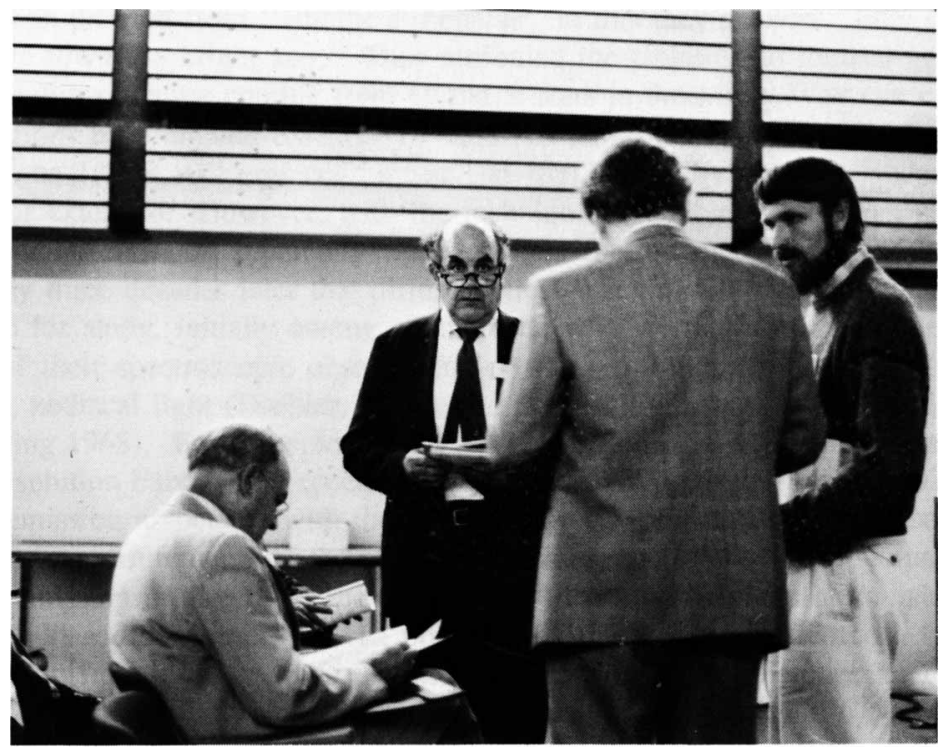

Preparing the following session: Guido Münch, Giovanni Fazio, Kalevi Mattila, and Christoph Leinert 